\title{
Next-to-leading order QCD corrections for single top-quark production in association with two jets
}

\author{
Stefan Mölbitz, ${ }^{1, *}$ Le Duc Ninh $\odot,{ }^{2, \dagger}$ and Peter Uwer ${ }^{1, *}$ \\ ${ }^{1}$ Humboldt-Universität zu Berlin, Institut für Physik, Newtonstraße 15, D-12489 Berlin, Germany \\ ${ }^{2}$ Institute For Interdisciplinary Research in Science and Education, ICISE, 590000 Quy Nhon, Vietnam
}

(Received 21 June 2019; published 21 January 2020)

\begin{abstract}
In this article we calculate the next-to-leading order (NLO) QCD corrections for single on-shell topquark production in association with two jets at proton-proton colliders. The $t W$ channel is assumed to be measured independently. The QCD corrections to the inclusive cross section are about 28\% (22\%) for top (antitop) quark production at the $13 \mathrm{TeV}$ LHC. Theoretical errors are dominated by scale uncertainties, which are found to be around 5\% at NLO. Results for various kinematical distributions are also provided using a well-motivated dynamical scale. The QCD corrections are found to have a nontrivial dependence on the phase space.
\end{abstract}

DOI: 10.1103/PhysRevD.101.016013

\section{INTRODUCTION}

Twenty-five years after its discovery many questions related to the top quark are still open, despite the tremendous progress made in recent years concerning the measurement of its mass and its interactions. Why is the top quark the only quark with a natural Yukawa coupling to the Higgs boson of order one? Why is it almost 35 times heavier than the next heavy quark, the $b$-quark? Are the top quark's weak interactions as in the Standard Model or does the top quark play a special role in the electroweak symmetry breaking as predicted in many extensions of the Standard Model?

The hadronic production of single top quarks allows one to shed light on these questions. In particular, singly produced top quarks provide an ideal laboratory to study the top-quark weak interactions. This is a major difference to top-quark pair production-with a roughly 3 times larger cross section, the dominant process for top-quark production in hadronic collisions-where the weak couplings are only accessible through the top-quark decay. Since most of the experimental efforts in recent years were devoted to pair production, the top quark's weak interactions are currently experimentally much less constrained through direct measurements than the top-quark

\footnotetext{
*moelbitz@physik.hu-berlin.de

†1dninh@ifirse.icise.vn

*Peter.Uwer@physik.hu-berlin.de
}

Published by the American Physical Society under the terms of the Creative Commons Attribution 4.0 International license. Further distribution of this work must maintain attribution to the author(s) and the published article's title, journal citation, and DOI. Funded by SCOAP ${ }^{3}$. strong interactions. A detailed study of single top-quark production offers the opportunity to fill this gap and to search for new physics. Furthermore, single top-quark production provides complementary information compared to top-quark pair production and allows studies not possible in top-quark pair production. While top quarks produced in pairs are to good approximation unpolarized (a tiny polarization is generated by QCD absorptive parts and weak corrections [1-3]), singly produced top quarks are highly polarized. Single topquark production presents thus a unique source of polarized top quarks which can be used for detailed tests of the $V-A$ structure of the coupling to the $W$ boson and to constrain potential new physics. In addition, single topquark production offers a direct handle to measure the Cabibbo-Kobayashi-Maskawa (CKM) matrix element $V_{t b}$-providing complementary information to indirect determinations based on unitarity.

Single top-quark production was first observed in proton-antiproton collisions at the Tevatron $[4,5]$. According to the virtuality of the $W$ boson occurring in the Born approximation, three different channels are distinguished: $s$-channel production with $p_{w}^{2}>0\left(p_{w}\right.$ denotes the four momentum of the $W$ boson), $t$-channel production with $p_{w}^{2}<0$, and the $t W$ channel where the $W$ boson occurs in the final state. The $t$-channel production is the dominant production process at the Tevatron and the LHC. At the Tevatron $s$-channel production is the second important channel. The $t W$ channel is suppressed at the Tevatron because of the limited collider energy of only $1.96 \mathrm{TeV}$. At the LHC the situation is reversed. The $t W$ channel represents the second important channel while the $s$ channel production is suppressed. Because of the challenging experimental environment and large backgrounds 
so far only evidence for $s$-channel production has been reported by the ATLAS experiment [6].

For all three production channels the next-to-leading order (NLO) QCD corrections have been calculated [7-15]. While initially only the inclusive cross sections have been analyzed, later works include also results for differential cross sections. In addition, the effects of the top-quark decay and the parton shower were analyzed [16-23]. Based on soft gluon resummation, approximate next-to-next-toleading order (NNLO) results have been published in Refs. [14,24-32]. An important step toward full NNLO results has been made for $t$-channel production in Refs. $[33,34]$ where NNLO results within the leading-color approximation are presented. Restricting the analysis to the leading-color contribution, the calculation of the NNLO corrections is significantly simplified, since the double box contributions, notoriously difficult to calculate, drop out. As a step beyond this approximation the reduction of the double-box topologies to master integrals has been performed in [35]. However, the occurring master integrals are still unknown, although progress toward their evaluation has been made in [36], where some of the integrals are studied as sample applications. Recently, the studies within the leading color approximation have been extended to include also the top-quark decay allowing one to study single top-quark production fully differentially at the level of the decay products [37].

Already at next-to-leading order, real corrections with an additional jet in the final state start to contribute. In fact, a detailed study shows that a significant fraction of single top-quark events is produced with additional jet activity. Demanding a minimal $p_{\perp}$ of $25 \mathrm{GeV}$, about $30 \%$ of singly produced top-quark events are produced in association with two jets. To make optimal use of the data collected at the LHC, precise predictions for single top-quark production in association with two jets are mandatory. For reliable theory predictions at least NLO QCD corrections are required. Furthermore, the NLO QCD corrections to single top-quark production in association with two jets contribute to single top-quark production at NNLO QCD and are thus required to extend the existing leading-color results. In this article, we present the NLO corrections to single top-quark production in association with two additional light jets. In principle, the NLO corrections can be produced with publicly available tools such as MadGraph5_aMC@NLO [38] or GoSam [39]. However, similar to single top-quark production the $t$-channel production needs to be separated from the $t W$ production, which requires one to remove some of the Feynman diagrams contributing to the full amplitude. Furthermore, the NLO corrections to single top-quark production in association with two additional jets contribute to the NNLO corrections to the inclusive single top-quark production. With this application in mind, we have decided to do the calculation by a direct evaluation of the Feynman diagrams and use Gosam only to partially cross-check the results. ${ }^{1}$

Let us mention that during the work on this project, similar results have been published in Ref. [40]. In this article, the authors work in the leading color approximation which is for the concrete process equivalent to the so-called structure function approximation. The key ingredient is that the QCD corrections are studied independently for the two incoming quark lines. At the same time this approximation gives also a clear separation from other single top-quark processes since interference terms are color suppressed. In addition to the fixed order calculation within the leadingcolor approximation, the results are further improved using the multi-scale improved NLO (MINLO) approach [41]. We consider the results presented in Ref. [40] as complementary to the ones presented here.

The outline of the article is as follows. In Sec. II we summarize the calculation and present some technical details. In Sec. III we describe the numerical input and present results for inclusive cross sections and various kinematical distributions. In addition we discuss the main uncertainties. We present a detailed discussion of the scale uncertainties and show the improvements using a dynamical scale. In Sec. IV conclusions are given. In the Appendix we show additional results for the production of antitop quarks.

\section{CALCULATION}

We consider on-shell production of a single top quark in association with two jets in proton-proton collisions. We work in the five flavor scheme. The bottom quark is thus treated as massless and considered as part of the proton. We neglect the generation mixing terms in the CKM matrix since these contributions are further suppressed by the parton distribution functions. Employing the unitarity of the CKM matrix this approximation is equivalent to replace the CKM matrix by the identity matrix. Using the identity matrix for the CKM matrix leads to a $C P$ invariant theory. It is thus sufficient to study top-quark production since the results for antitop-quark production can be obtained from $C P$ invariance.

Care must be taken to separate top-quark production in association with two jets from the $t W$ channel with subsequent hadronic decay of the $W$-boson. The latter process leads to the same final state but is measured separately by the experiments. This issue is well known from single top-quark production. In leading order the

\footnotetext{
${ }^{1}$ When we started the project we expected that the handwritten code might be faster than publicly available code generators for NLO amplitudes, which could be an advantage when the contribution is calculated as part of an NNLO calculation. However, benchmarks we did at the end when the project was completed showed that GoSam and MadGraph5_aMc@NLO produce code which is comparably fast if not even faster.
} 


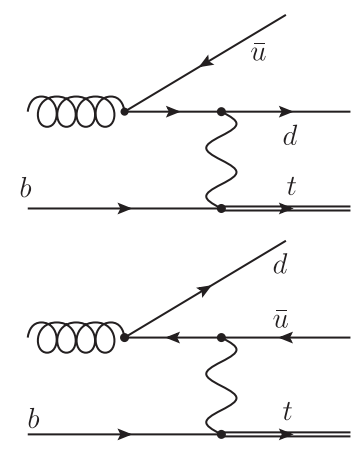

(a) $t j j$

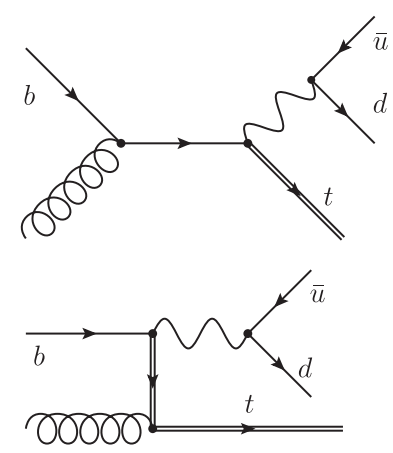

(b) $t W$

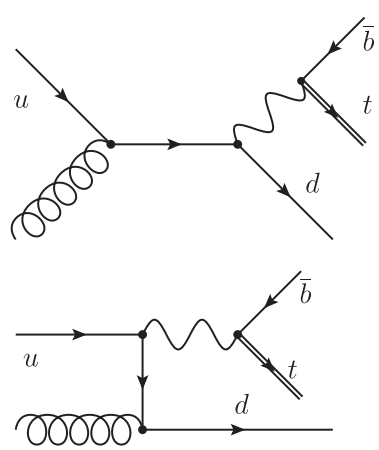

(c) $t j j$

FIG. 1. Representative LO diagrams classified into $t j j$ and $t W$ production channels.

interference term between the two contributions vanishes and the individual contributions are gauge invariant. We assume that these contributions are small in next-to-leading order-in particular, when experimental cuts to separate the $t W$ channel are applied. For a similar discussion we refer to Ref. [40] where this approximation is used to justify the structure function approximation. Whenever we refer to the $t j j$ channel in the following, the contribution from on-shell $t W$ production and subsequent decay is removed. More details will be given below.

\section{A. Leading order}

At LO, all subprocesses can be classified into two groups: with or without $\bar{U}_{i} D_{i}$ in the final state, where $i=1,2$ for the first two generations and capital letters are used to denote the generic up- or down-type light quarks. All subprocesses without $\bar{U}_{i} D_{i}$ in the final state belong to the $t j j$ channel.

For the remaining subprocesses, a light quark can come either from the initial gluon [see Fig. 1(a)] or from an intermediate $W$ boson [see Fig. 1(b)]. The former diagrams belong to the $t j j$ channel, while the latter diagrams belong to the $t W$ channel. As mentioned before the interference between the two contributions vanishes due to the different color structures. Moreover, since each group is separately gauge invariant, the $t W$-channel diagrams can be completely removed. Note that similar diagrams but with the $W \rightarrow t \bar{b}$ vertex [see Fig. 1(c)] are classified as part of the tjj channel.

Technically, all amplitudes can be obtained via the crossing of one subprocess, say $b u \rightarrow t d g$. For the crossed channel $g b \rightarrow t \bar{u} d$, care must be taken to select only the diagrams which belong to the $t j j$ channel as explained above.

\section{B. Next-to-leading order QCD}

NLO QCD contributions include virtual and real-emission corrections. The real-emission processes have one additional parton in the final state. Because of this additional QCD emission, color factors of the $t j j$ and $t W$ amplitudes become more involved and allow interference between the two contributions. Moreover, the $t \bar{t}$ channel, where a top quark decays into a $W$ boson and a bottom quark, can lead to the same final state of $t+3$ jets. Furthermore, the interference between the $t j j$ and the $t \bar{t}$ channels is also nonvanishing. Similar to what has been done for single top-quark production, these contributions have to be treated separately to account for the experimental analysis in which the three processes are analyzed independently. This can be done in a gauge invariant way by performing a pole expansion and keeping only nonresonant contributions. Assuming that experimental cuts will highly suppress these contributions, this corresponds in practice to dropping resonant diagrams such as Fig. 2(c). Care must be taken when additional radiation can lead in general to off-shell contributions and when on-shell contributions are only generated in certain phase space regions such as in Fig. 2(b). In this case the on-shell contributions can be extracted using the soft-gluon approximation [42-45] in combination with the complex mass scheme [46-48]. It is well known that because of soft-collinear factorization real and virtual corrections cancel each other in the soft limit for

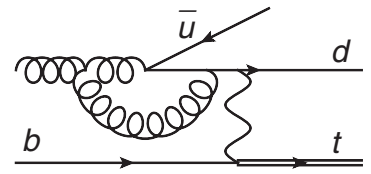

(a) $t j j$

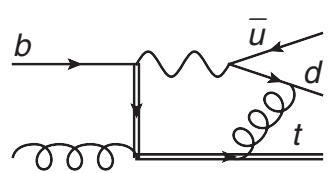

(b) $t j j$

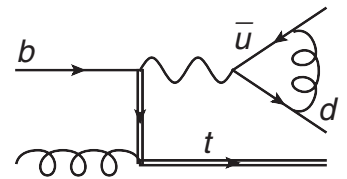

(c) $t W$

FIG. 2. Representative one-loop diagrams classified into $t j j$ and $t W$ production channels. 


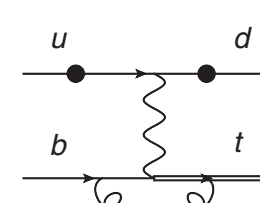

(a)

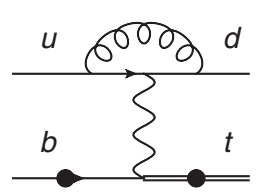

(b)

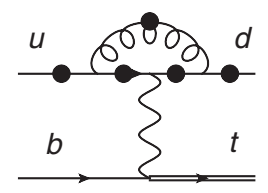

(c)

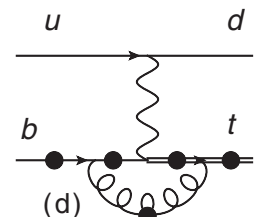

(d)

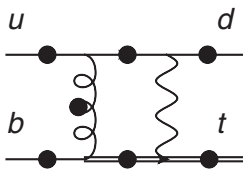

(e)

FIG. 3. Five gauge-invariant groups contributing to the virtual correction of the subprocess $b u \rightarrow t d g$. The dots represent possible positions for a gluon emission. Only representative diagrams are shown. Similar contributions for the other subprocesses can be obtained via crossings as for the LO case.

sufficiently inclusive quantities (see e.g., Ref. [49] where this is shown for a concrete example). To approximate the nonresonant part of this contribution, we have combined the respective virtual corrections with the corresponding real corrections approximated through the $I$-operator within the Catani-Seymour subtraction method. Even without applying further experimental cuts to suppress the $t W$ channel, we find that this contribution gives only a tiny correction at the sub-permille level and it can safely be dropped within the uncertainties of the final result. For the final results presented in Sec. III and Appendix, this correction is nevertheless included.

We note that all Feynman diagrams for every subprocess can be classified in gauge-invariant groups. At LO, there are always two quark lines connected by a $W$ boson exchange. The two gauge-invariant groups correspond therefore to two cases: the additional gluon is connected either to the heavy-quark line (i.e., with the top quark) or to the light-quark line. When a virtual gluon exchange is added, five gauge-invariant groups arise. The virtual gluon can be attached exclusively to the light-quark line as in Figs. 3(b) and 3(c) or to the heavy-quark line as in Figs. 3(a) and 3(d). This makes four gauge-invariant groups. The remaining group corresponds to the case when there is color exchange between the two quark lines as in Fig. 3(e) [see also Fig. 2(b)]. This group contains oneloop five-point integrals and is thus the most complicated one to calculate. Numerically, this group of diagrams is strongly suppressed and gives only a tiny contribution to the full result.

We have performed two independent calculations. For both calculations, the dipole subtraction method [50,51] is used. The real-emission amplitudes are IR divergent in soft and collinear limits. These singularities have to be regularized and subtracted using subtraction terms before being integrated over the phase space in four dimensions. In this step, the $t W$ and $t \bar{t}$ resonant diagrams are removed; hence interference effects with the $t j j$ channels are neglected. The subtraction terms are built from the reduced $2 \rightarrow 3$ amplitudes keeping only the $t j j$ diagrams as for the LO contribution. These subtraction terms have to be added back in the form of integrated dipole contributions called $P K_{t j j}$ and $I_{t j j}$ operators. The IR singularities in these operators are canceled by the corresponding ones in the parton distribution function (PDF) counterterms and in the virtual corrections.

The analytic results have been implemented in two different computer codes, one written in $\mathrm{C}++$, the other in FORTRAN. Extensive cross-checks have been done. We have compared results at the amplitude level as well as results for the integrated cross sections and distributions. Within the numerical uncertainties perfect agreements between the two calculations have been obtained. Details about these comparisons are provided in Ref. [52].

Before presenting the results we would like to provide further details of the calculation. In the $\mathrm{C}++$ program, the scalar one-loop integrals are calc QCD loop [53] and FF [54]. The $N$-point tensor integrals are reduced to scalar integrals using the Passarino-Veltman method [55] for $N \leq 3$ and the tensor-reduction library PJFRY [56] for the cases $N=4,5$. The library PJFRY uses the methods presented in Ref. [57]. This calculation has been cross-checked with a reduction using Ref. [58] and with GoSam [59]. The amplitudes for the real corrections are obtained using MadGraph5_aMC@NLO [38]. The phase space integration has been done using the Monte Carlo integrator vEGAS [60].

In the FORTRAN program, scalar one-loop integrals are calculated using an in-house library Loopints based on the techniques of Refs. [61-63]. $\mathrm{N}$-point tensor integrals are reduced to scalar integrals using the Passarino-Veltman method [55] for $N \leq 4$ and Ref. [64] for $N=5$. LoopI uses by default double precision, but will automatically switch to quadruple precision if numerical instabilities occur in the tensor reduction. Helicity amplitudes are generated using the programs FormCalc [65], FeynArts [66], and MadGraph-v4 [67] which uses HELAS routines [68]. The $I$ operators required in the Catani-Seymour subtraction algorithm are implemented with the help of AutoDipole [69]. The integrator BASES [70] is used for the phase-space integration.

\section{PHENOMENOLOGICAL RESULTS}

For the input values we use

$$
\begin{aligned}
G_{F} & =1.16638 \times 10^{-5} \mathrm{GeV}^{-2}, \quad m_{W}=80.385 \mathrm{GeV}, \\
m_{t} & =(173.21 \pm 0.87) \mathrm{GeV} .
\end{aligned}
$$

The masses of all light quarks, i.e., all but the top-quark mass, are set to zero. Partons are combined into jets using 
TABLE I. Inclusive cross sections with PDF, $\alpha_{s}$ and $m_{t}$ uncertainties calculated at LO.

\begin{tabular}{lcccc}
\hline \hline$\sigma_{\mathrm{LO}}$ & {$[\mathrm{pb}]$} & $(\delta \sigma)_{\mathrm{PDF}}[\%]$ & $(\delta \sigma)_{\alpha_{s}}[\%]$ & $(\delta \sigma)_{m_{t}}[\%]$ \\
\hline$\sigma_{t}$ & 22.2 & \pm 1.7 & \pm 1.6 & \pm 0.8 \\
$\sigma_{\bar{t}}$ & 14.7 & \pm 2.1 & \pm 1.6 & \pm 0.9 \\
$\sigma_{t+\bar{t}}$ & 36.9 & \pm 1.9 & \pm 1.6 & \pm 0.8 \\
\hline \hline
\end{tabular}

the anti- $k_{t}$ algorithm [71] with the radius $R$ set to $R=0.4$. We treat the top quark as a stable particle and do not include the top-quark decay. We assume that the top quark is always tagged and do not apply the jet algorithm to the top-quark momentum. The momentum of the jet containing the top quark is thus identified with the top-quark momentum. In addition, we impose the following cuts on the remaining jets:

$$
p_{T, j}>25 \mathrm{GeV}, \quad\left|\eta_{j}\right|<2.5,
$$

where $p_{T, j}$ denotes the transverse momentum and $\eta_{j}$ the pseudorapidity of the jet. For the PDFs, we use the PDF4LHC15_NLO_100_PDFAS set [72-78] via the library LHAPDF6 [79]. For the QCD coupling constant $\alpha_{s}$ the value provided by the PDF set, corresponding to $\alpha_{s}\left(m_{Z}\right)=0.118$ $\left(m_{Z}=91.1876 \mathrm{GeV}\right)$ for the chosen PDF, is taken. The same PDF set is used for both LO and NLO results. We produce results for the LHC running at a center-of-mass energy of $\sqrt{s}=13 \mathrm{TeV}$.

\section{A. Inclusive cross sections}

Using the aforementioned setup we find in leading order for $\sqrt{s}=13 \mathrm{TeV}$ for the production of a single (anti)top quark in association with two additional jets the cross sections shown in Table I. The cross section for topquark production is about 1.5 times larger than the cross section for antitop-quark production. We also show in Table I the uncertainties due to an imperfect knowledge of the PDFs, the QCD coupling constant $\alpha_{s}$, and the topquark mass. The PDF and $\alpha_{s}$ uncertainties are calculated as defined in Ref. [72]. Estimating the PDF uncertainties using the error PDFs provided by the PDF set, we find an uncertainty of $1.7 \%$ for top-quark production and a slightly larger uncertainty of $2.1 \%$ for antitop-quark production. For the uncertainties due to $\alpha_{s}$ we find in both cases an uncertainty of $1.6 \%$. The uncertainty due to a variation of the top-quark mass within the bounds allowed by the uncertainty in Eq. (3.1) leads to an effect of $0.8 \%-0.9 \%$ - consistent with the naive expectation based on the mass dependence of $t$-channel single topquark production [80]. In conclusion the numerical input is thus sufficiently well known to allow precise predictions of the cross section.

In Table II results for the cross section in NLO accuracy are given. The quoted values are for $\mu=\mu_{F}=\mu_{R}=m_{t}$ where $\mu_{F}$ denotes the factorization scale and $\mu_{R}$ the renormalization scale. As the central scale the top-quark mass is used. The NLO corrections enhance the cross section by $22 \%$ in case of antitop-quark production and almost 30\% in case of top-quark production. In Table II we have also included the effects due to a change of the central scale $\mu$ by a factor of 2 up and down. The uncertainty is estimated by varying independently the two scales $\mu_{F}$ and $\mu_{R}$ as $n \mu_{0} / 2$ with $n=1,2,4$ and $\mu_{0}=m_{t}$. The constraint $1 / 2 \leq \mu_{R} / \mu_{F} \leq 2$ is used to avoid "extrem" scale ratios and associated potentially large logarithms. This limits the number of possible scale choices to seven ("seven-point method"). To estimate the uncertainty we determine the maximal and minimal values for the cross section. As far as the leading order cross sections are concerned, this leads to an uncertainty of about $\pm 10 \%$. The NLO corrections are thus significantly larger than the range covered by the leading-order scale uncertainties, showing that the scale variation does not provide a reliable uncertainty estimate for the specific cross section. As expected, the inclusion of the NLO corrections lead to a significant reduction of the scale uncertainty by roughly a factor of 2 . Compared to the aforementioned uncertainties related to uncertainties of the PDFs, $\alpha_{s}$ and $m_{t}$, missing higher orders thus provide the dominant source of uncertainty.

In Fig. 4 the scale dependence of the inclusive cross sections at LO and NLO for single top-quark production in association with at least two additional jets is shown for the case $\mu_{F}=\mu_{R}=\mu$. The two-jet exclusive cross section at NLO, where any additional jet activity is vetoed, is also shown. The difference between the inclusive and the twojet exclusive cross sections gives the contribution of the three-jet events. Note that the latter is only predicted in LO accuracy. The corresponding plot for antiquark production shows a similar behavior; see Fig. 12 in Appendix. As can be seen in Fig. 4 even in NLO the cross sections show a scale dependence typical for a leading-order calculation.

TABLE II. Inclusive cross sections with scale uncertainties. The central scale is $\mu_{0}=m_{t}$. Numbers in the parentheses are statistical errors on the last digits.

\begin{tabular}{|c|c|c|c|c|c|c|c|c|}
\hline$\sigma_{\mathrm{LO}}$ & [pb] & $(\delta \sigma)_{\text {scale }}[\%]$ & [pb] & $\sigma_{\mathrm{NLO}}$ & [pb] & $(\delta \sigma)_{\text {scale }}[\%]$ & [pb] & $K$ \\
\hline$\sigma_{t}$ & $22.195(2)$ & $\begin{array}{l}+10.5 \\
-8.7\end{array}$ & $\begin{array}{l}+2.3 \\
-1.9\end{array}$ & $\sigma_{t}$ & $28.307(8)$ & $\begin{array}{l}+5.8 \\
-4.5\end{array}$ & $\begin{array}{l}+1.6 \\
-1.3\end{array}$ & 1.28 \\
\hline$\sigma_{\bar{t}}$ & $14.671(1)$ & $\begin{array}{l}+10.5 \\
-87\end{array}$ & +1.5 & $\sigma_{\bar{t}}$ & $17.856(7)$ & $\begin{array}{l}+4.1 \\
-38\end{array}$ & $\begin{array}{l}1.7 \\
+0.7\end{array}$ & 1.22 \\
\hline$\sigma_{t+\bar{t}}$ & $36.866(2)$ & $\begin{array}{l}-8.7 \\
+10.5 \\
-8.7\end{array}$ & $\begin{array}{l}-1.3 \\
+3.9 \\
-3.2\end{array}$ & $\sigma_{t+\bar{t}}$ & $46.163(11)$ & $\begin{array}{l}-5.0 \\
+5.1 \\
-4.2\end{array}$ & $\begin{array}{l}-0.1 \\
+2.4 \\
-1.9\end{array}$ & 1.25 \\
\hline
\end{tabular}




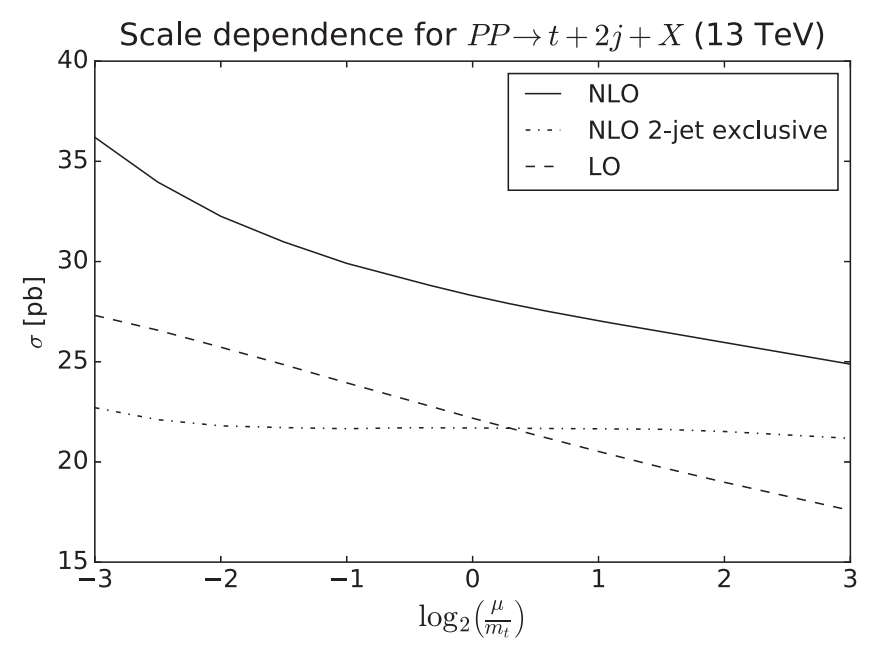

FIG. 4. Scale dependence of the inclusive cross sections $\sigma_{\mathrm{LO}}$, $\sigma_{\mathrm{NLO}}$ and of the two-jet exclusive cross section $\sigma_{\mathrm{NLO}}^{2 \mathrm{jet} \text { exc }}$. Factorization and renormalization scales are set equal, i.e., $\mu_{F}=\mu_{R}=\mu$.

In particular, the scale dependence is not flat. This is because at NLO new channels occur, including in particular subprocesses with two gluons. These new channels are numerically large and dominate the scale dependence. To illustrate this effect, the two-jet cross section where additional jet activity is vetoed is given. The veto suppresses the contribution from the new channels and leads to a significantly improved scale dependence of the two-jet exclusive cross section. However, this does not necessarily mean that the theoretical predictions for this observable are more precise. It is well known that the jet veto introduces an additional scale and can lead to additional uncertainties in particular in differential distributions. This is because the veto scale can lead to large logarithmic corrections which may spoil the convergence

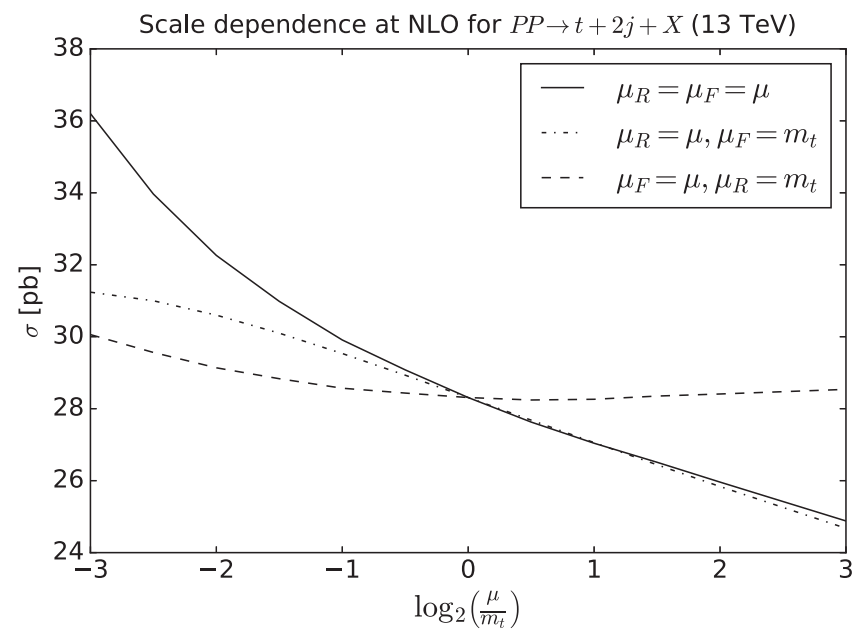

FIG. 5. Dependence of the inclusive cross sections $\sigma_{\mathrm{NLO}}$ on the individual scales $\mu_{F}$ and $\mu_{R}$.
TABLE III. Cross sections with scale uncertainties at different proton-proton colliding energies.

\begin{tabular}{lcccc}
\hline \hline$\sqrt{s}[\mathrm{TeV}]$ & $\sigma_{\mathrm{LO}}^{t}[\mathrm{pb}]$ & $\sigma_{\mathrm{NLO}}^{t}[\mathrm{pb}]$ & $\sigma_{\mathrm{LO}}^{\bar{t}}[\mathrm{pb}]$ & $\sigma_{\mathrm{NLO}}^{\bar{t}}[\mathrm{pb}]$ \\
\hline 7 & $7.4_{-0.8}^{+0.9}$ & $8.9_{-0.3}^{+0.3}$ & $4.0_{-0.5}^{+0.5}$ & $4.8_{-0.2}^{+0.2}$ \\
8 & $9.6_{-1.0}^{+1.1}$ & $11.6_{-0.4}^{+0.4}$ & $5.4_{-0.6}^{+0.7}$ & $6.5_{-0.3}^{+0.3}$ \\
13 & $22.2_{-1.9}^{+2.3}$ & $28.3_{-1.3}^{+1.6}$ & $14.7_{-1.3}^{+1.5}$ & $17.9_{-0.7}^{+0.7}$ \\
14 & $25.0_{-2.2}^{+2.6}$ & $32.2_{-1.5}^{+1.5}$ & $16.9_{-1.5}^{+1.8}$ & $20.7_{-0.8}^{+0.8}$ \\
\hline \hline
\end{tabular}

of the perturbative expansion if not resummed. For more details we refer to Ref. [81].

In Fig. 5 we show results where only one of the two scales $\mu_{F}, \mu_{R}$ is changed while the other is kept fixed. In the range $-1<\log _{2}\left(\mu / m_{t}\right)<3$ the renormalization scale gives the dominant contribution to the scale dependence. This is consistent with the aforementioned observation that the scale dependence is dominated by new channels which occur for the first time in NLO. Only at rather low scales does the factorization scale become important.

In Table III we show the cross section for different collider energies. In the case of antitop-quark production the $K$-factor is only weakly dependent on the collider energy. In contrast, for top-quark production a significant rise of the $K$-factor can be observed. As a consequence also the ratio of the two cross sections depends on the collider energy. At high energies the number of produced antitop quarks increases with respect to the number of produced top quarks. This is because top-quark and antitop-quark production probe different PDFs with a different energy dependence.

\section{B. Kinematical distributions}

For the evaluation of the inclusive cross section we used a fixed renormalization and factorization scale. While this is appropriate for the total cross section which is dominated by events with moderate momentum transfer, this is no longer true when distributions at high momentum transfer are studied. In the latter case numerically rather different energy scales can occur which may lead to large logarithmic corrections invalidating the naive use of perturbation theory. It is well known that in such cases a dynamical scale often improves the situation. For the process at hand, we use

TABLE IV. LO cross sections with a dynamical scale using different values for the constant $c_{d}$. For comparison, the result for the fixed scale is also given.

\begin{tabular}{lccccccc}
\hline \hline$c_{d}$ & 2 & 1 & $1 / 2$ & $1 / 4$ & $1 / 8$ & $1 / 16$ & $\mu=m_{t}$ \\
\hline$\sigma_{\mathrm{LO}}^{t}[\mathrm{pb}]$ & 18.38 & 19.83 & 21.40 & 23.06 & 24.74 & 26.26 & 22.20 \\
$\sigma_{\mathrm{LO}}^{t}[\mathrm{pb}]$ & 12.12 & 13.12 & 14.20 & 15.34 & 16.49 & 17.56 & 14.67 \\
\hline \hline
\end{tabular}


TABLE V. Inclusive cross sections with scale uncertainties. The central scale is $\mu_{\mathrm{dyn}}^{0}$. Numbers in the parentheses are statistical errors on the last digit. Scale uncertainties are calculated using the three-point $\left(\mu_{\mathrm{dyn}}^{0} / 2, \mu_{\mathrm{dyn}}^{0}, 2 \mu_{\mathrm{dyn}}^{0}\right)$ method.

\begin{tabular}{|c|c|c|c|c|c|c|c|c|}
\hline$\sigma_{\mathrm{LO}}$ & [pb] & $(\delta \sigma)_{\text {scale }}[\%]$ & {$[\mathrm{pb}]$} & $\sigma_{\mathrm{NLO}}$ & [pb] & $(\delta \sigma)_{\text {scale }}[\%]$ & [pb] & $K$ \\
\hline$\sigma_{t}$ & $21.407(2)$ & $\begin{array}{l}+7.7 \% \\
-7.3 \%\end{array}$ & $\begin{array}{l}+1.7 \\
{ }_{-1.6}\end{array}$ & $\sigma_{t}$ & $27.14(1)$ & $\begin{array}{l}+4.7 \% \\
-3.9 \%\end{array}$ & $\begin{array}{l}+1.3 \\
-1.1\end{array}$ & 1.27 \\
\hline$\sigma_{\bar{t}}$ & 14.197(1) & $\begin{array}{l}+8.0 \% \\
-7.6 \%\end{array}$ & $\begin{array}{l}-1.1 \\
+1.1\end{array}$ & $\sigma_{\bar{t}}$ & $17.23(2)$ & $\begin{array}{l}-3.4 \% \\
-3.1 \%\end{array}$ & $\begin{array}{l}-1.1 \\
+0.6 \\
-0.5\end{array}$ & 1.21 \\
\hline$\sigma_{t+\bar{t}}$ & $35.604(2)$ & $\begin{array}{l}+7.9 \% \\
-7.4 \% \\
\end{array}$ & $\begin{array}{l}+2.8 \\
-2.6 \\
\end{array}$ & $\sigma_{t+\bar{t}}$ & $44.37(2)$ & $\begin{array}{l}+4.2 \% \\
-3.6 \%\end{array}$ & $\begin{array}{l}+1.9 \\
-1.6\end{array}$ & 1.25 \\
\hline
\end{tabular}

$$
\mu_{\mathrm{dyn}}=c_{d}\left(m_{t}+p_{T, t}+\sum_{i \in \text { partons }} p_{T, i}\right)
$$

as the dynamical scale, where $c_{d}$ is a constant which still needs to be fixed to a certain value. The above scale choice may be seen as a modification of $H_{T}$ often used. The topquark mass occurring in the dynamical scale prevents the scale from becoming too small for low energies, since in this case the top-quark mass provides a cutoff and should be the relevant energy scale.

To find a reasonable value for the constant $c_{d}$, we require that the inclusive cross section matches the one calculated with $\mu_{F}=\mu_{R}=m_{t}$. In Table IV LO results are provided, varying $c_{d}$ from $1 / 16$ to 2 . The results show that $c_{d}=1 / 2$ is a good choice and reproduces roughly the results obtained with the fixed scale. We will therefore use hereafter this value to define the central scale $\mu_{\text {dyn }}^{0}$.

In Table V we present NLO cross sections together with the associated scale uncertainties using the dynamical scale with $c_{d}=1 / 2$. The scale uncertainties are estimated using $\mu_{F}=\mu_{R}=\mu_{\mathrm{dyn}}^{0}$ and varying the dynamical scale by a factor of 2 up and down. The results for the inclusive cross section are in good agreement with the results obtained for a fixed scale evaluated at $\mu=m_{t}$. Note that, different from the scale uncertainties presented in Table II where the seven-point method is used, the values in Table $\mathrm{V}$ are obtained using the three-point method where we identify $\mu_{F}=\mu_{R}$ and the two scales are varied together. This is done to be consistent with the distributions, where we use the three-point method to reduce the required computing time. The uncertainties agree reasonably well with the ones in Table II.

In Fig. 6 we compare for the case of the $p_{T^{-}}$ distribution of the top quark the two different scale choices. The left-hand plot shows the result obtained with a fixed scale as used in the previous section. The right-hand plot shows the same distribution using the dynamical scale. Using the fixed scale the absolute value of the corrections increases for large transverse momentum signaling a breakdown of perturbation theory due to the appearance of the aforementioned large logarithmic corrections. This is also partially reflected in the increasing uncertainty estimated through scale variation. Using the dynamical scale instead, we find a much improved behavior. Even at high momentum transfer, the corrections amount only to $-20 \%$. As anticipated, using the dynamical scale leads thus to a significant improvement of the perturbative expansion. We stress that the scale choice affects only mildly the NLO corrections. This is illustrated in the upper plot of Fig. 7. Independent of whether a dynamic scale or a fixed scale is chosen, the
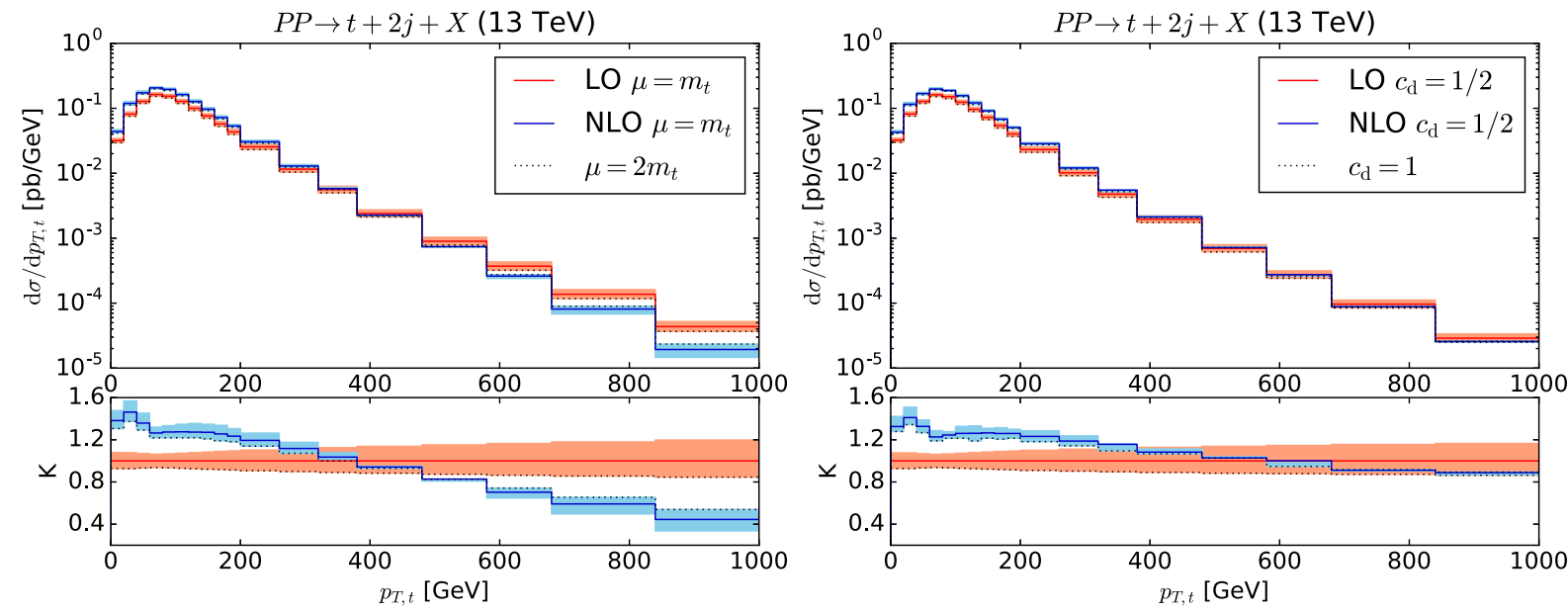

FIG. 6. Distributions of the transverse momentum of the top quark with fixed scale $\mu=m_{t}$ (left) and with dynamical scale $\mu=\mu_{\mathrm{dyn}}^{0}$ (right). 


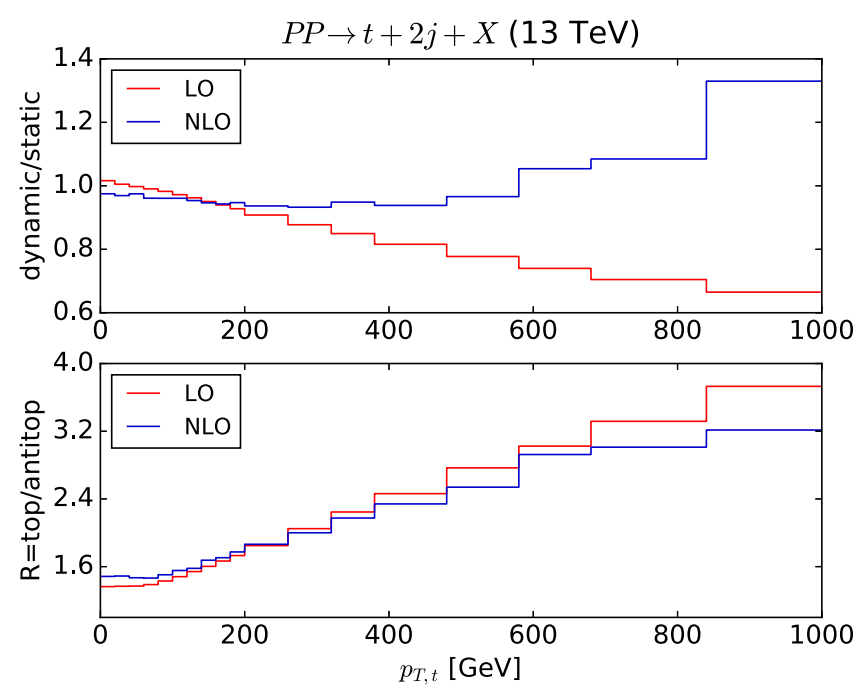

FIG. 7. Comparison of predictions using a dynamical scale with predictions using a fixed scale (upper plot) for the top-quark production. Ratio between the top-quark and antitop-quark production (lower plot).

NLO corrections are roughly the same. The ratio of the two predictions is very close to one. Only beyond $800 \mathrm{GeV}$ is this no longer true. In case the dynamical scale is used, the LO predictions give thus a better prescription of the full result.

The lower plot of Fig. 7 shows the ratio of the cross section for top-quark production and the cross section for antitop-quark production. The red curve shows the LO result while the blue curve is the NLO one. The two curves are very close to each other. However, one can see that the ratio is highly $p_{T}$ dependent. At low $p_{T}$ we recover roughly the factor of 1.5 observed for the total cross section. With increasing $p_{T}$ the ratio increases. This information can be used in the experimental analysis to compare for example top-quark and antitop-quark tagging efficiencies.

In Fig. 8 we show the transverse momentum distribution for the top quark, the first jet, and the second jet. The jets are ordered in $p_{T}$ with the first jet having the largest transverse momentum. With the exception of the low momentum region, one observes a flat $K$-factor amounting to positive corrections of the order $20 \%-$ $30 \%$. Most of the jets have a transverse momentum below $100 \mathrm{GeV}$. In the case of the second jet, the fraction of jets having a transverse momentum above $100 \mathrm{GeV}$ is below $10 \%$. The $p_{T}$-distribution of the top-quark jet peaks at about $75 \mathrm{GeV}$. The $p_{T}$-distribution of the leading light jet is narrower compared to the top-quark distribution and peaks at a slightly smaller $p_{T}$ value. The $p_{T^{-}}$ distribution of the second light jet is a steeply falling function. The lower end is set by the minimal $p_{T}$ required by the jet definition. In the case of the $p_{T^{-}}$ distribution of the top quark the NLO corrections lead to
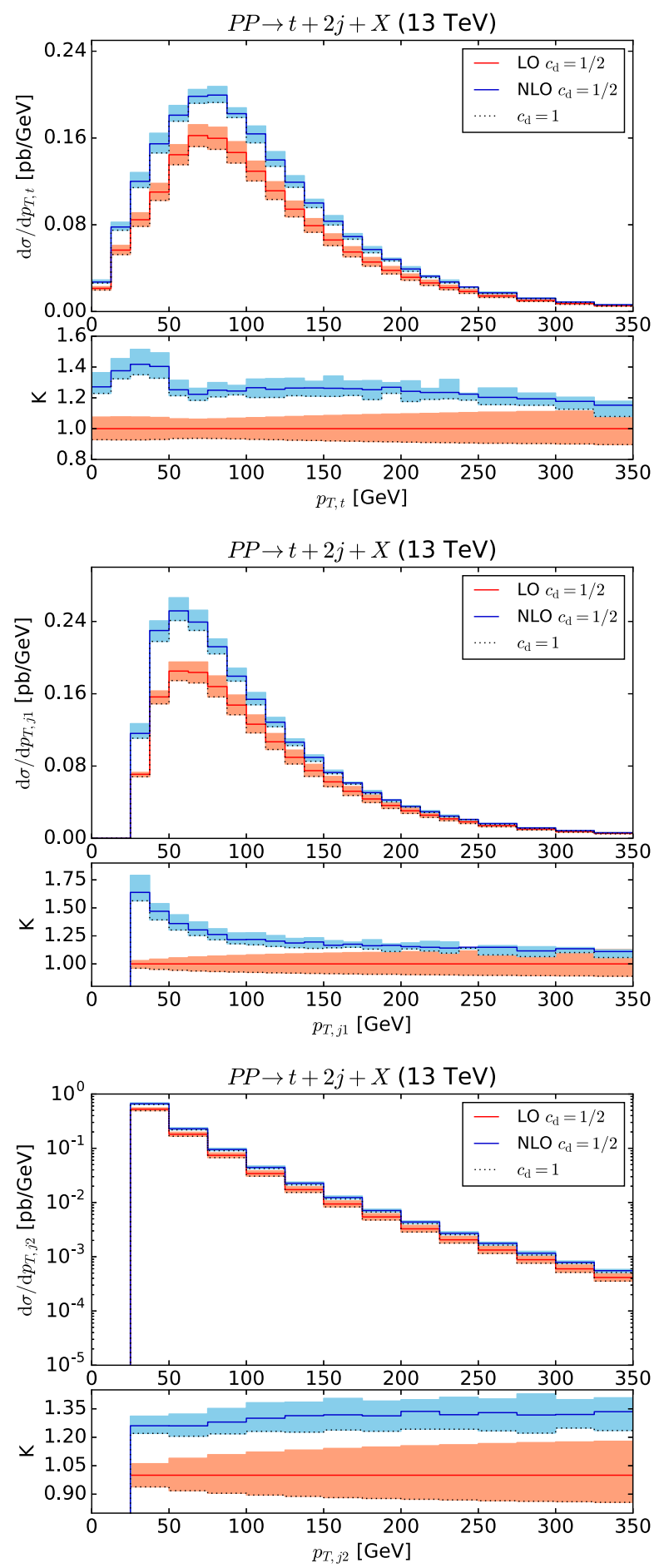

FIG. 8. Distributions of the transverse momentum of the top quark (top), the first jet (middle), and the second jet (bottom).

an enhancement below $50 \mathrm{GeV}$. This is an effect of the real corrections. The total transverse momentum must add up to zero. In leading order, the $p_{T}$ of the top quark must be compensated by the total $p_{T}$ of the two additional jets with each having at least a minimal $p_{T}$ of $25 \mathrm{GeV}$ to pass the cuts. A top-quark transverse momentum below 

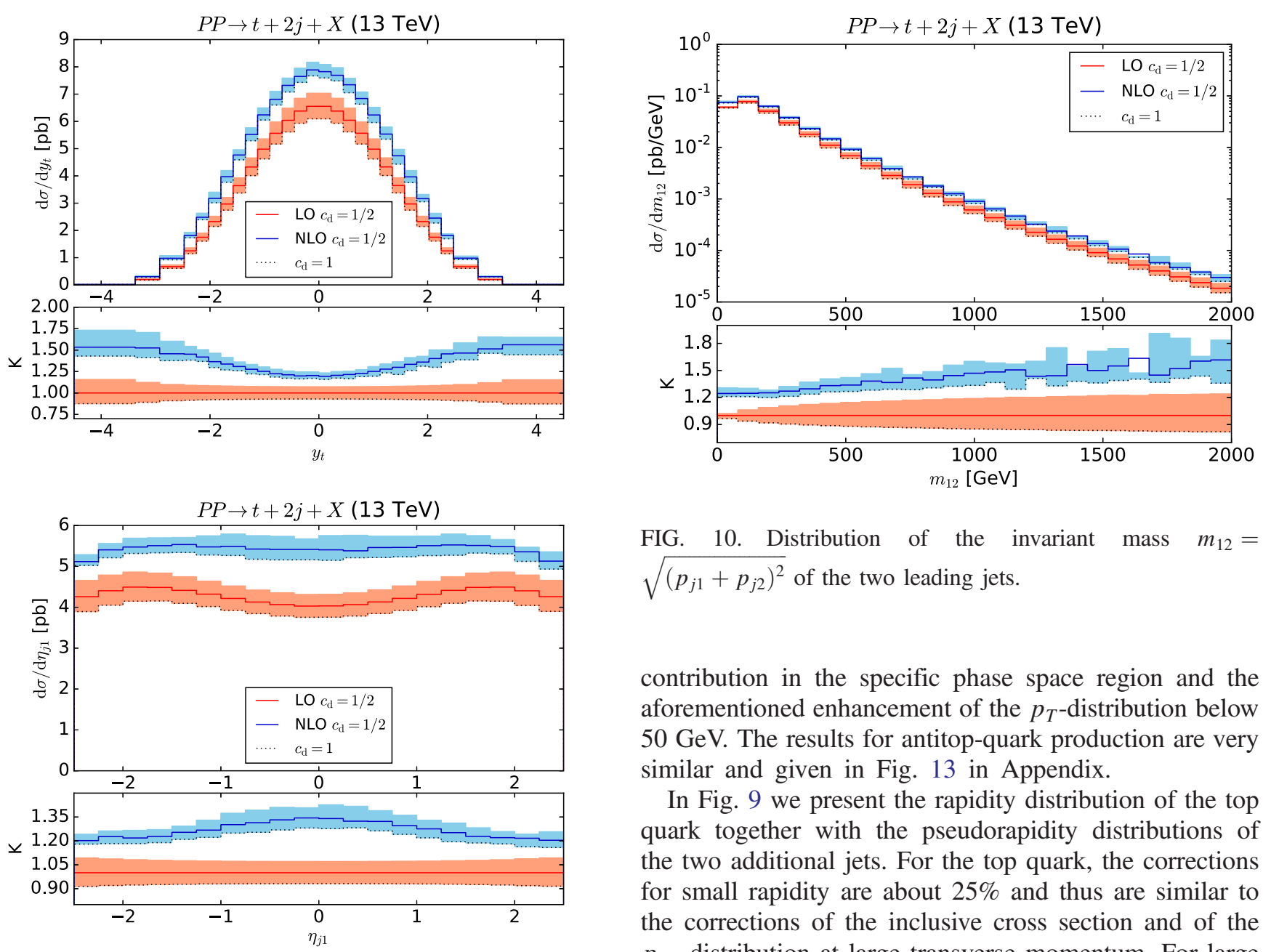

FIG. 10. Distribution of the invariant mass $m_{12}=$ $\sqrt{\left(p_{j 1}+p_{j 2}\right)^{2}}$ of the two leading jets.

contribution in the specific phase space region and the aforementioned enhancement of the $p_{T}$-distribution below $50 \mathrm{GeV}$. The results for antitop-quark production are very similar and given in Fig. 13 in Appendix.

In Fig. 9 we present the rapidity distribution of the top quark together with the pseudorapidity distributions of the two additional jets. For the top quark, the corrections for small rapidity are about $25 \%$ and thus are similar to the corrections of the inclusive cross section and of the $p_{T, t}$-distribution at large transverse momentum. For large

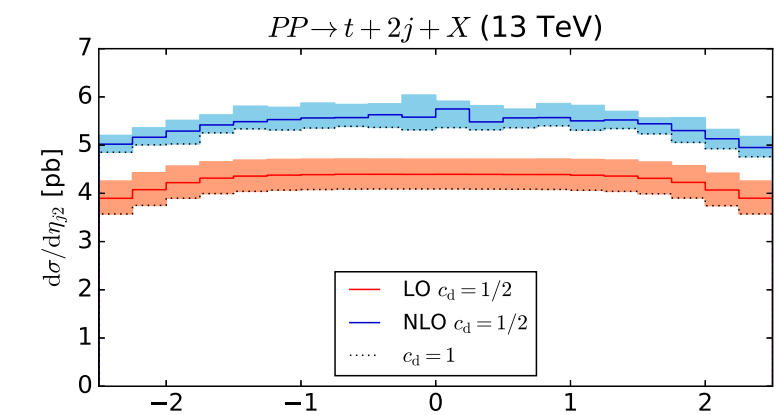
$\left|y_{t}\right|$ the corrections increase, however, and are of the order of $50 \%$. The origin of this effect is similar to the effect observed in the $p_{T}$-distribution of the top quark and is again a consequence of the real corrections. A large top-quark rapidity corresponds to a small value of the top-quark transverse momentum which requires in leading order again a very special phase space configuration for the two additional jets. The additional jet in the real corrections extends the available phase space and leads thus to a positive correction to the cross section. This is also reflected in the scale dependence. Since the effect is due to the real corrections, the results show a large scale dependence. As far as the NLO results for the pseudorapidity distribution of the two light jets are concerned, the results look very similar. Both jets show a rather flat distribution with a slight enhancement for small $|\eta|$. In the case of the leading jet, a dip occurring for $\eta_{j 1}=0$ in leading order is mostly washed out by the NLO corrections. At NLO only a minor depletion is visible for small $|\eta|$. The origin of this effect has been traced back to the three-jet event contribution of the quark-gluon induced channels (quark $\neq b$ ), with the $u g$ subprocess being the dominant contribution. This is consistent with the observation that the NLO scale 

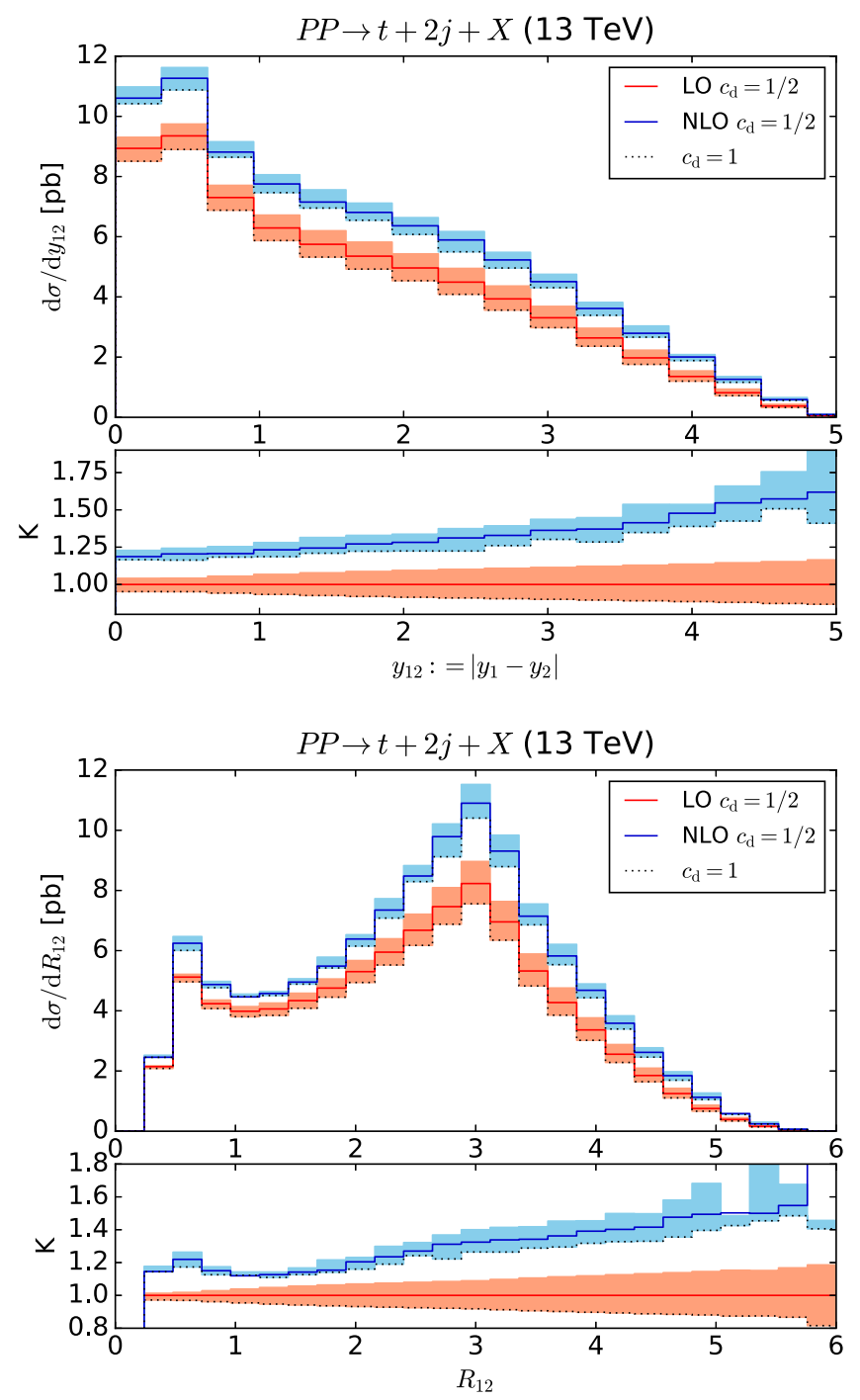

FIG. 11. Distributions of the rapidity separation (top) and the $R$ separation (bottom) of the two leading jets.

uncertainties are large around $\eta_{j 1}=0$. Similar results for the antitop-quark production are shown in Fig. 14 in Appendix. We observe that there the dip in the LO $\eta_{j 1}$ distribution is much less pronounced compared to the top-quark production. This is most probably due to the difference in the $u$ and $d$ PDFs.

In Fig. 10 the distribution of the invariant mass of the two light jets is shown. For small $m_{12}=\sqrt{\left(p_{j 1}+p_{j 2}\right)^{2}}$ one observes again positive corrections of about $20 \%$. The corrections increase with increasing $m_{12}$. At the same time the scale uncertainty-although reduced compared to the leading order results-becomes larger. The increasing corrections are due to the real corrections. We have checked that the virtual corrections ( $I$ operator included) are negative and are not responsible for that behavior. Similar results for the antitop-quark production are provided in Fig. 15 in Appendix.
To quantify the spatial separation between the two leading jets we define

$$
\Delta y_{12}=\left|y_{1}-y_{2}\right|
$$

and

$$
\Delta R_{12}=\sqrt{\left(y_{1}-y_{2}\right)^{2}+\left(\phi_{1}-\phi_{2}\right)^{2}} .
$$

In Fig. 11 the distributions of $y_{12}$ and $R_{12}$ are shown. As one can see from the upper plot, a small rapidity difference between the two leading jets is the preferred configuration. The lower plot of Fig. 11 shows the distributions of the distance in the $y-\phi$ plane. The LO and NLO $\Delta R_{12}$ distributions peak around $\pi$, which corresponds, for small rapidity differences, to the configuration that the two jets are back-to-back. There is a second less pronounced peak around 0.5 . In this case the two jets recoil against the top quark. Note that $\Delta R_{12}$ must be larger than 0.4 because of the jet definition. For moderate $\Delta y_{12}$ and $\Delta R_{12}$ the NLO corrections to the $\Delta y_{12}$ and $\Delta R_{12}$ distributions tend to be slightly larger than for the inclusive cross section. The corrections increase for large $\Delta y_{12}$ and large $\Delta R_{12}$. As can be seen in Fig. 11 the scale uncertainties increase together with the size of the corrections. Again this is an effect of the real corrections. The corresponding distributions for the antitop-quark production show a similar behavior and are given in Fig. 16 in Appendix.

\section{CONCLUSIONS}

We have presented a calculation of the NLO QCD corrections for single on-shell top-quark production in association with two jets at the LHC. It is assumed that the $t W$ production mode is measured separately. At LO, the interference between the $t j j$ and the $t W$ channels vanishes because of different color structures. At NLO, additional QCD radiation introduces interference effects between $t j j$ and $t W$, and also with the $t \bar{t}$ production with one top quark decaying into three jets. However, these production modes peak in different phase-space regions; hence interference effects are expected to be very small, in particular when experimental cuts to separate the different channels are applied. We have checked that within the soft-gluon approximation the contribution is indeed tiny. With this assumption, the $t j j$ contribution can be measured independently.

Using inclusive cuts of $p_{T, j}>25 \mathrm{GeV},\left|\eta_{j}\right|<2.5$, and the anti- $k_{t}$ algorithm with a radius $R=0.4$ to define jets, the NLO QCD corrections for the cross section at $13 \mathrm{TeV}$ are about $28 \%$ (22\%) for top (antitop) quark production. The theoretical uncertainties are dominated by missing higher order contributions, which are estimated, using a variation of the renormalization and 
factorization scales, to be about $5 \%$ at NLO. Uncertainties due to an imperfect knowledge of the PDFs and of the strong coupling constant are about $2 \%$ at LO.

Further predictions for various kinematical distributions have been provided. Using a well-motivated dynamical scale choice for the renormalization and factorization scales, in most cases moderate $K$-factors are observed, showing similar corrections as the inclusive cross section. However, the QCD corrections have a nontrivial dependence on the phase space leading to large corrections in specific phase-space regions. For example, for the $p_{T}$ distribution of the top quark, the correction is about $+40 \%$ in the region of $p_{T} \leq 50 \mathrm{GeV}$, and then drops to about $+20 \%$ for $50<p_{T}<300 \mathrm{GeV}$ before decreasing steadily with high energies. Corresponding results for the antitop-quark production have also been presented, thereby allowing for comparisons between the two production modes.

The results presented here provide one of the missing building blocks toward the next-to-next-to-leading order QCD corrections for single top-quark production beyond the leading color approximation.

\section{ACKNOWLEDGMENTS}

The work has been partly supported by the German Ministry of Education and Research under Contract No. 05H15KHCAA. The work of L.D. N. is funded by the Vietnam National Foundation for Science and Technology Development (NAFOSTED) under Grant No. 103.01-2017.78.

\section{APPENDIX: RESULTS FOR ANTITOP-QUARK PRODUCTION}

In this Appendix, results for antitop-quark production are provided. Namely, the scale dependence is shown in

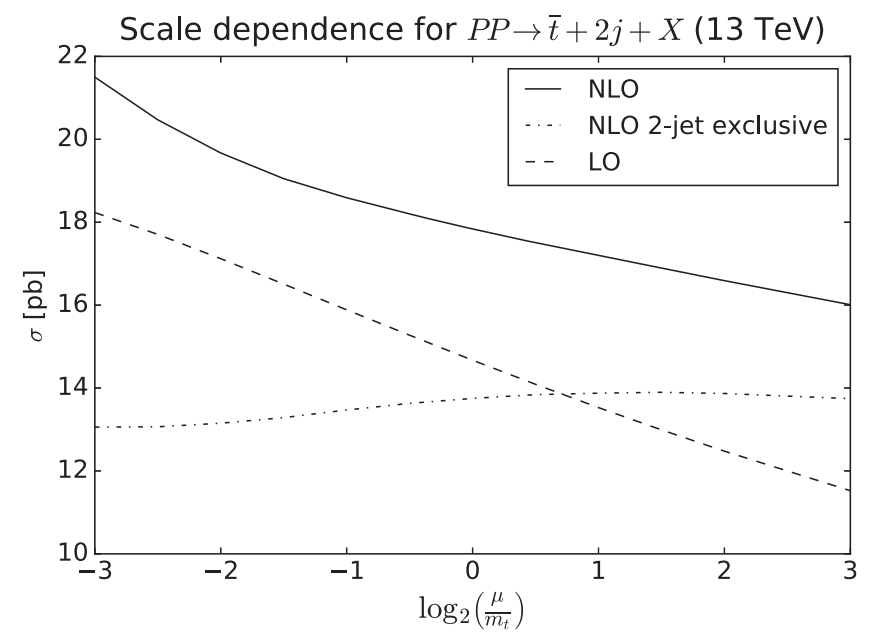

FIG. 12. Same as Fig. 4 but for antitop-quark production.
Fig. 12; the transverse momentum distributions of the antitop quark and of the two leading jets are displayed in Fig. 13; the rapidity distribution of the antitop-quark and the pseudorapidity distributions of the two leading jets are in Fig. 14; the invariant mass distribution of the two leading jets is shown in Fig. 15; and finally the
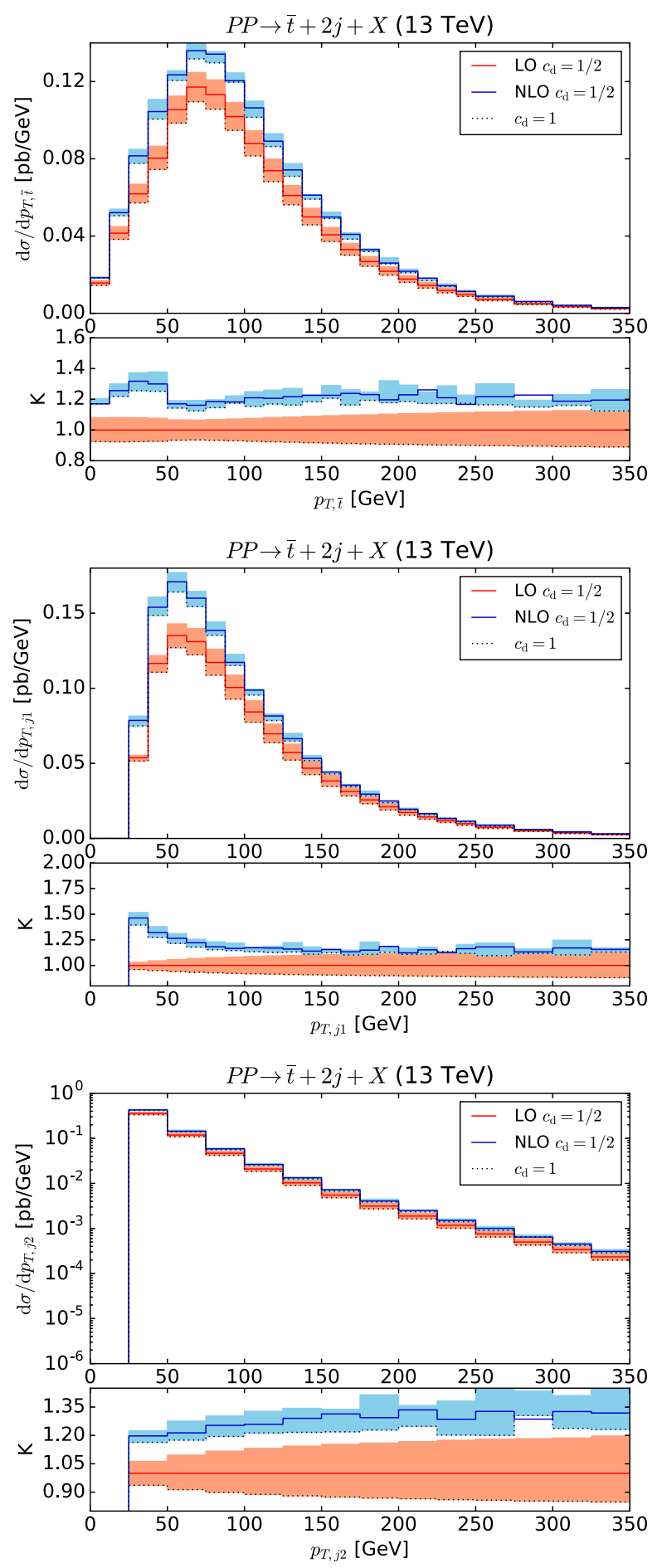

FIG. 13. Same as Fig. 8 but for antitop-quark production. 

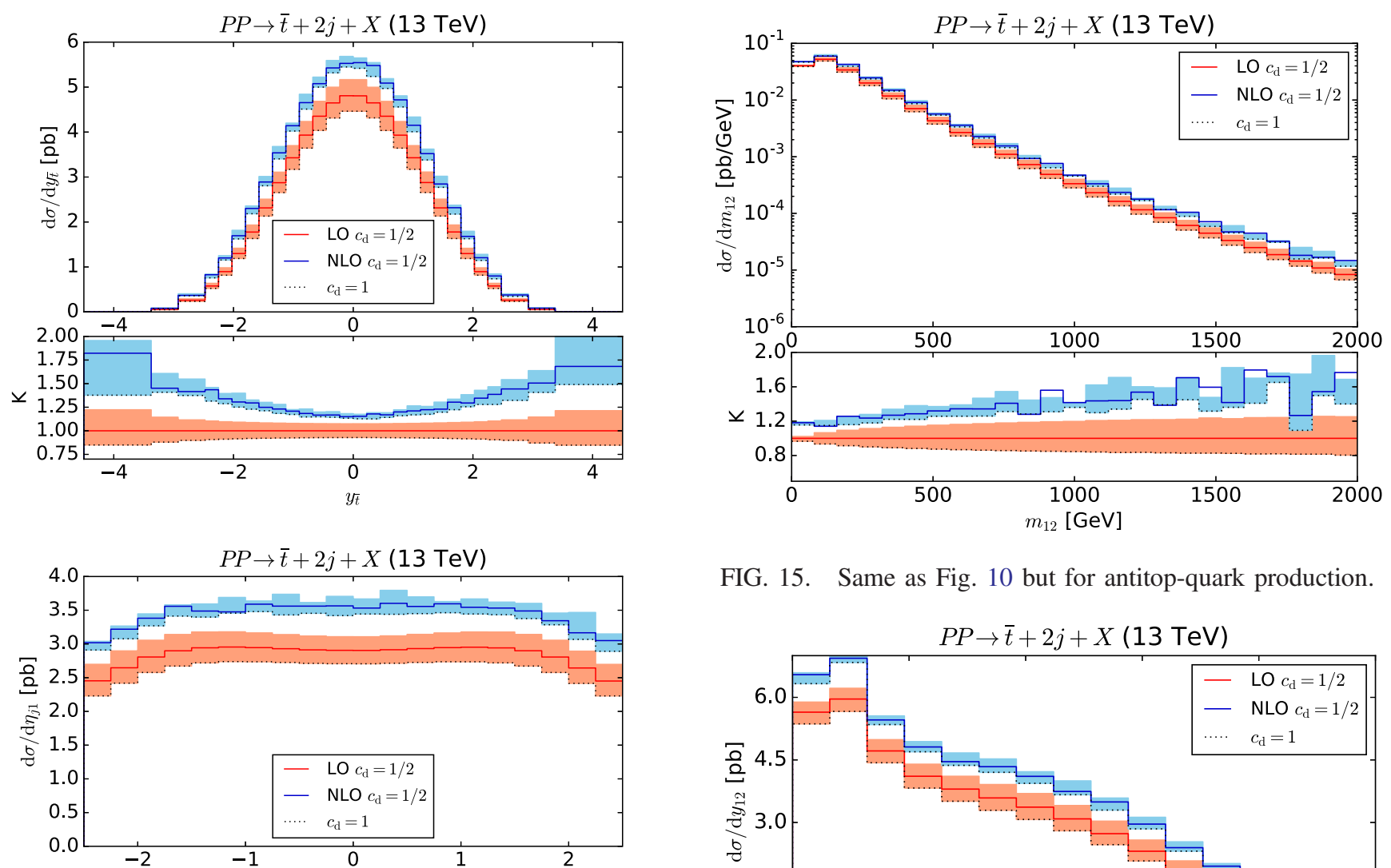

FIG. 15. Same as Fig. 10 but for antitop-quark production.
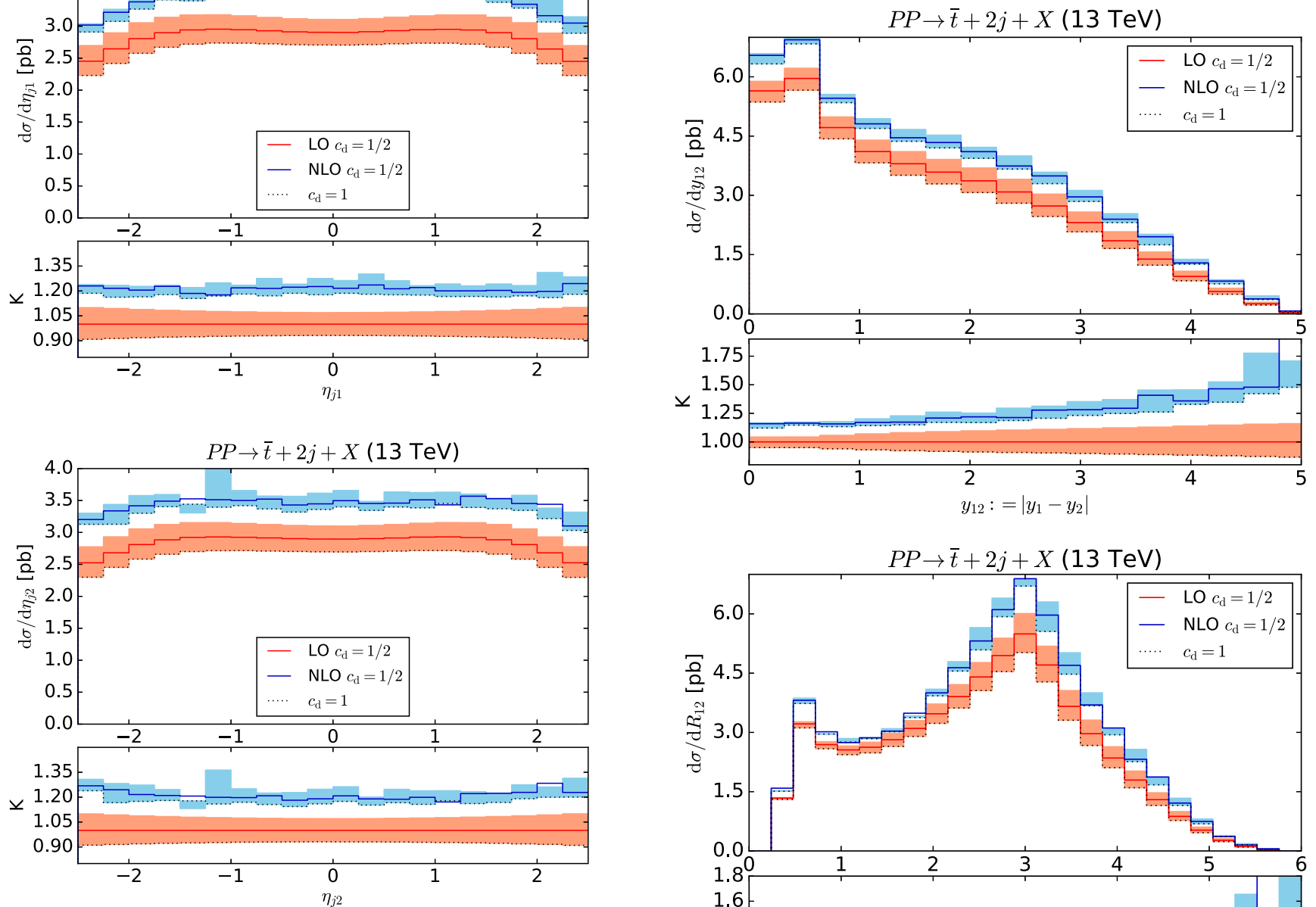

FIG. 14. Same as Fig. 9 but for antitop-quark production.

distributions of the rapidity and $R$ separation between the two leading jets as defined in Eq. (3.4) and Eq. (3.5), respectively, are presented in Fig. 16.

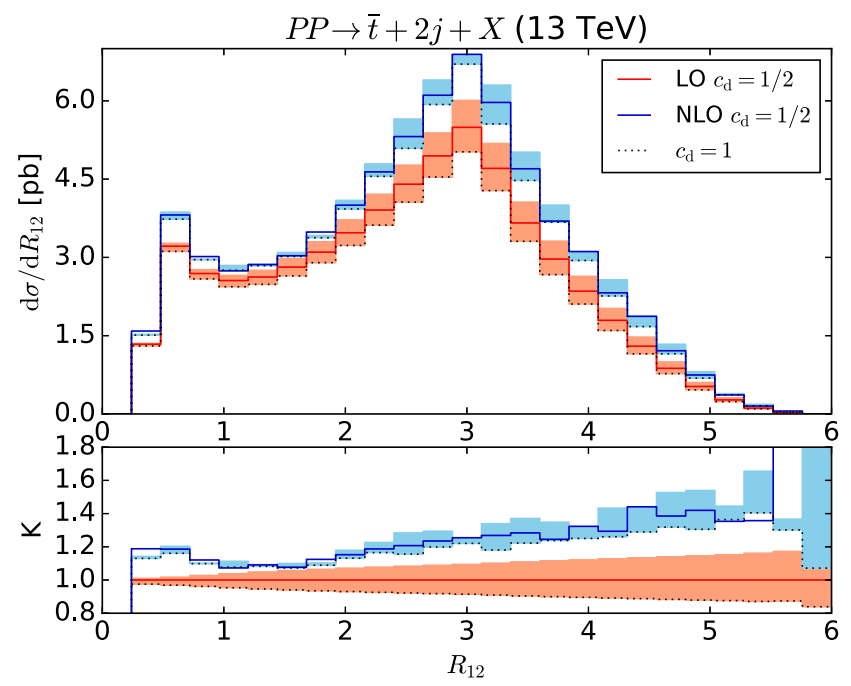

FIG. 16. Same as Fig. 11 but for antitop-quark production. 
[1] W. Bernreuther, A. Brandenburg, and P. Uwer, Transverse polarization of top quark pairs at the Tevatron and the large hadron collider, Phys. Lett. B 368, 153 (1996).

[2] W. G. D. Dharmaratna and G. R. Goldstein, Gluon fusion as a source for massive quark polarization, Phys. Rev. D 41, 1731 (1990).

[3] W. Bernreuther and Z.-G. Si, Distributions and correlations for top quark pair production and decay at the Tevatron and LHC, Nucl. Phys. B837, 90 (2010).

[4] T. Aaltonen et al. (CDF Collaboration), First Observation of Electroweak Single Top Quark Production, Phys. Rev. Lett. 103, 092002 (2009).

[5] V. M. Abazov et al. (D0 Collaboration), Observation of Single Top Quark Production, Phys. Rev. Lett. 103, 092001 (2009).

[6] G. Aad et al. (ATLAS Collaboration), Evidence for single top-quark production in the $s$-channel in proton-proton collisions at $\sqrt{s}=8 \mathrm{TeV}$ with the ATLAS detector using the matrix element method, Phys. Lett. B 756, 228 (2016).

[7] G. Bordes and B. van Eijk, Calculating QCD corrections to single top production in Hadronic interactions, Nucl. Phys. B435, 23 (1995).

[8] T. Stelzer, Z. Sullivan, and S. Willenbrock, Single top quark production via W-Gluon fusion at next-to-leading order, Phys. Rev. D 56, 5919 (1997).

[9] T. Stelzer, Z. Sullivan, and S. Willenbrock, Single top quark production at Hadron colliders, Phys. Rev. D 58, 094021 (1998).

[10] B. Harris, E. Laenen, L. Phaf, Z. Sullivan, and S. Weinzierl, The fully differential single top quark cross-section in next to leading order QCD, Phys. Rev. D 66, 054024 (2002).

[11] Z. Sullivan, Understanding single-top-quark production and jets at Hadron colliders, Phys. Rev. D 70, 114012 (2004).

[12] Z. Sullivan, Angular correlations in single-top-quark and Wjj production at next-to-leading order, Phys. Rev. D 72, 094034 (2005).

[13] W. Giele, S. Keller, and E. Laenen, QCD corrections to $W$ Boson plus heavy quark production at the Tevatron, Phys. Lett. B 372, 141 (1996).

[14] S. Zhu, Next-to-leading order QCD corrections to $b g \rightarrow$ $t W^{-}$at the CERN Large Hadron Collider, Phys. Lett. B 524, 283 (2002).

[15] M. Smith and S. Willenbrock, QCD and Yukawa corrections to single top quark production via $q \bar{q} \rightarrow t \bar{b}$, Phys. Rev. D 54, 6696 (1996).

[16] J. Campbell, R. Ellis, and F. Tramontano, Single top production and decay at next-to-leading order, Phys. Rev. D 70, 094012 (2004).

[17] Q. Cao and C.-P. Yuan, Single top quark production and decay at next-to-leading order in Hadron collision, Phys. Rev. D 71, 054022 (2005).

[18] Q. Cao, R. Schwienhorst, J. Benitez, R. Brock, and C.-P. Yuan, Next-to-leading order corrections to single top quark production and decay at the Tevatron: 2. $t$-channel process, Phys. Rev. D 72, 094027 (2005).

[19] S. Frixione, E. Laenen, P. Motylinski, and B. Webber, Single-top production in MC@NLO, J. High Energy Phys. 03 (2006) 092.
[20] J. Campbell and F. Tramontano, Next-to-leading order corrections to $\mathrm{Wt}$ production and decay, Nucl. Phys. B726, 109 (2005).

[21] S. Frixione, E. Laenen, P. Motylinski, B. R. Webber, and C. D. White, Single-top Hadroproduction in association with a W Boson, J. High Energy Phys. 07 (2008) 029.

[22] E. Re, Single-top Wt-channel production matched with Parton showers using the PowHeg method, Eur. Phys. J. C 71, 1547 (2011).

[23] Q. Cao, R. Schwienhorst, and C.-P. Yuan, Next-to-leading order corrections to single top quark production and decay at Tevatron. 1. s-channel process, Phys. Rev. D 71, 054023 (2005).

[24] S. Mrenna and C. Yuan, Effects of QCD resummation on $W^{+} h$ and $t \bar{b}$ production at the Tevatron, Phys. Lett. B 416, 200 (1998).

[25] N. Kidonakis, Single top production at the Tevatron: Threshold resummation and finite-order soft gluon corrections, Phys. Rev. D 74, 114012 (2006).

[26] N. Kidonakis, Higher-order soft gluon corrections in single top quark production at the LHC, Phys. Rev. D 75, 071501 (2007).

[27] N. Kidonakis, Two-loop soft anomalous dimensions for single top quark associated production with a $\mathrm{W}^{-}$or $\mathrm{H}^{-}$, Phys. Rev. D 82, 054018 (2010).

[28] N. Kidonakis, Next-to-next-to-leading soft-gluon corrections for the top quark cross section and transverse momentum distribution, Phys. Rev. D 82, 114030 (2010).

[29] N. Kidonakis, Next-to-next-to-leading-order collinear and soft gluon corrections for $t$-channel single top quark production, Phys. Rev. D 83, 091503 (2011).

[30] Q.-H. Cao, P. Sun, B. Yan, C. P. Yuan, and F. Yuan, Transverse momentum resummation for $t$-channel single top quark production at the LHC, Phys. Rev. D 98, 054032 (2018).

[31] P. Sun, B. Yan, and C. P. Yuan, Transverse momentum resummation for $s$-channel single top quark production at the LHC, Phys. Rev. D 99, 034008 (2019).

[32] Q.-H. Cao, P. Sun, B. Yan, C. P. Yuan, and F. Yuan, Soft gluon resummation in $t$-channel single top quark production at the LHC, arXiv:1902.09336.

[33] M. Brucherseifer, F. Caola, and K. Melnikov, On the NNLO QCD corrections to single-top production at the LHC, Phys. Lett. B 736, 58 (2014).

[34] E. L. Berger, J. Gao, C. P. Yuan, and H. X. Zhu, NNLO QCD corrections to t-channel single top-quark production and decay, Phys. Rev. D 94, 071501 (2016).

[35] M. Assadsolimani, P. Kant, B. Tausk, and P. Uwer, Calculation of two-loop QCD corrections for hadronic single top-quark production in the $\mathrm{t}$ channel, Phys. Rev. D 90, 114024 (2014).

[36] C. Meyer, Transforming differential equations of multi-loop Feynman integrals into canonical form, J. High Energy Phys. 04 (2017) 006.

[37] E. L. Berger, J. Gao, and H. X. Zhu, Differential distributions for t-channel single top-quark production and decay at next-to-next-to-leading order in QCD, J. High Energy Phys. 11 (2017) 158.

[38] J. Alwall, R. Frederix, S. Frixione, V. Hirschi, F. Maltoni, O. Mattelaer, H.-S. Shao, T. Stelzer, P. Torrielli, and M. Zaro, 
The automated computation of tree-level and next-to-leading order differential cross sections, and their matching to parton shower simulations, J. High Energy Phys. 07 (2014) 079.

[39] G. Cullen et al., GoSAM-2.0: A tool for automated oneloop calculations within the Standard Model and beyond, Eur. Phys. J. C 74, 3001 (2014).

[40] S. Carrazza, R. Frederix, K. Hamilton, and G. Zanderighi, MINLO t-channel single-top plus jet, J. High Energy Phys. 09 (2018) 108.

[41] K. Hamilton, P. Nason, and G. Zanderighi, MINLO: Multiscale improved NLO, J. High Energy Phys. 10 (2012) 155.

[42] V.S. Fadin, V. A. Khoze, and A. D. Martin, Interference radiative phenomena in the production of heavy unstable particles, Phys. Rev. D 49, 2247 (1994).

[43] K. Melnikov and O. I. Yakovlev, Final state interaction in the production of heavy unstable particles, Nucl. Phys. B471, 90 (1996).

[44] W. Beenakker, A. P. Chapovsky, and F. A. Berends, Nonfactorizable corrections to $\mathrm{W}$ pair production: Methods and analytic results, Nucl. Phys. B508, 17 (1997).

[45] S. Dittmaier, A. Huss, and C. Schwinn, Mixed QCDelectroweak $\mathrm{O}\left(\alpha_{s} \alpha\right)$ corrections to Drell-Yan processes in the resonance region: Pole approximation and nonfactorizable corrections, Nucl. Phys. B885, 318 (2014).

[46] A. Denner, S. Dittmaier, M. Roth, and D. Wackeroth, Predictions for all processes $e^{+} e^{-} \rightarrow 4$ fermions $+\gamma$, Nucl. Phys. B560, 33 (1999).

[47] A. Denner, S. Dittmaier, M. Roth, and L. H. Wieders, Electroweak corrections to charged-current $e^{+} e^{-} \rightarrow 4$ fermion processes: Technical details and further results, Nucl. Phys. B724, 247 (2005).

[48] M. Nowakowski and A. Pilaftsis, On gauge invariance of Breit-Wigner propagators, Z. Phys. C 60, 121 (1993).

[49] W. Bernreuther, P. Galler, C. Mellein, Z. G. Si, and P. Uwer, Production of heavy Higgs bosons and decay into top quarks at the LHC, Phys. Rev. D 93, 034032 (2016).

[50] S. Catani and M. Seymour, A general algorithm for calculating jet cross-sections in NLO QCD, Nucl. Phys. B485, 291 (1997).

[51] S. Catani, S. Dittmaier, M. H. Seymour, and Z. Trocsanyi, The dipole formalism for next-to-leading order QCD calculations with massive partons, Nucl. Phys. B627, 189 (2002).

[52] S. Mölbitz, QCD Korrekturen zur Erzeugung von einzelnen top-quarks in assoziation mit zwei jets (in German), Ph.D. thesis, Humboldt-Universität zu Berlin, MathematischNaturwissenschaftliche Fakultät, 2019, https://doi.org/ $10.18452 / 19654$.

[53] R. K. Ellis and G. Zanderighi, Scalar one-loop integrals for QCD, J. High Energy Phys. 02 (2008) 002.

[54] G. J. van Oldenborgh, FF: A package to evaluate one loop Feynman diagrams, Comput. Phys. Commun. 66, 1 (1991).

[55] G. Passarino and M. J. G. Veltman, One loop corrections for $e^{+} e^{-}$annihilation into $\mu^{+} \mu^{-}$in the Weinberg model, Nucl. Phys. B160, 151 (1979).

[56] J. Fleischer, T. Riemann, and V. Yundin, One-loop tensor feynman integral reduction with signed minors, J. Phys. Conf. Ser. 368, 012057 (2012).
[57] J. Fleischer and T. Riemann, A complete algebraic reduction of one-loop tensor Feynman integrals, Phys. Rev. D 83, 073004 (2011).

[58] W. T. Giele and E. W. N. Glover, A calculational formalism for one loop integrals, J. High Energy Phys. 04 (2004) 029.

[59] G. Cullen et al., GOSAM-2.0: A tool for automated oneloop calculations within the Standard Model and beyond, Eur. Phys. J. C 74, 3001 (2014).

[60] G. P. Lepage, A new algorithm for adaptive multidimensional integration, J. Comput. Phys. 27, 192 (1978).

[61] G. 't Hooft and M. J. G. Veltman, Scalar one loop integrals, Nucl. Phys. B153, 365 (1979).

[62] D. T. Nhung and L. D. Ninh, D0C: A code to calculate scalar one-loop four-point integrals with complex masses, Comput. Phys. Commun. 180, 2258 (2009).

[63] A. Denner and S. Dittmaier, Scalar one-loop 4-point integrals, Nucl. Phys. B844, 199 (2011).

[64] A. Denner and S. Dittmaier, Reduction schemes for oneloop tensor integrals, Nucl. Phys. B734, 62 (2006).

[65] T. Hahn and M. Perez-Victoria, Automatized one loop calculations in four-dimensions and D-dimensions, Comput. Phys. Commun. 118, 153 (1999).

[66] T. Hahn, Generating Feynman diagrams and amplitudes with FeynArts 3, Comput. Phys. Commun. 140, 418 (2001).

[67] T. Stelzer and W. F. Long, Automatic generation of tree level helicity amplitudes, Comput. Phys. Commun. 81, 357 (1994).

[68] H. Murayama, I. Watanabe, and K. Hagiwara, HELAS: HELicity amplitude subroutines for feynman diagram evaluations, Report No. KEK-91-11, 1992, https://lib-extopc .kek.jp/preprints/PDF/1991/9124/9124011.pdf.

[69] K. Hasegawa, S. Moch, and P. Uwer, AutoDipole: Automated generation of dipole subtraction terms, Comput. Phys. Commun. 181, 1802 (2010).

[70] S. Kawabata, A new version of the multidimensional integration and event generation package BASES/SPRING, Comput. Phys. Commun. 88, 309 (1995).

[71] M. Cacciari, G. P. Salam, and G. Soyez, The anti- $k_{t}$ jet clustering algorithm, J. High Energy Phys. 04 (2008) 063.

[72] J. Butterworth et al., PDF4LHC recommendations for LHC Run II, J. Phys. G 43, 023001 (2016).

[73] S. Dulat, T.-J. Hou, J. Gao, M. Guzzi, J. Huston, P. Nadolsky, J. Pumplin, C. Schmidt, D. Stump, and C.-P. Yuan, New parton distribution functions from a global analysis of quantum chromodynamics, Phys. Rev. D 93, 033006 (2016).

[74] L. A. Harland-Lang, A. D. Martin, P. Motylinski, and R. S. Thorne, Parton distributions in the LHC era: MMHT 2014 PDFs, Eur. Phys. J. C 75, 204 (2015).

[75] R. D. Ball et al. (NNPDF Collaboration), Parton distributions for the LHC Run II, J. High Energy Phys. 04 (2015) 040.

[76] J. Gao and P. Nadolsky, A meta-analysis of parton distribution functions, J. High Energy Phys. 07 (2014) 035.

[77] S. Carrazza, S. Forte, Z. Kassabov, J. I. Latorre, and J. Rojo, An unbiased Hessian representation for Monte Carlo PDFs, Eur. Phys. J. C 75, 369 (2015).

[78] G. Watt and R. S. Thorne, Study of Monte Carlo approach to experimental uncertainty propagation with MSTW 2008 PDFs, J. High Energy Phys. 08 (2012) 052. 
[79] A. Buckley, J. Ferrando, S. Lloyd, K. Nordström, B. Page, M. Rüfenacht, M. Schönherr, and G. Watt, LHAPDF6: parton density access in the LHC precision era, Eur. Phys. J. C 75, 132 (2015).

[80] P. Kant, O. M. Kind, T. Kintscher, T. Lohse, T. Martini, S. Mölbitz, P. Rieck, and P. Uwer, HATHOR for single top-quark production: Updated predictions and uncertainty estimates for single top-quark production in hadronic collisions, Comput. Phys. Commun. 191, 74 (2015).

[81] I. W. Stewart and F. J. Tackmann, Theory uncertainties for Higgs and other searches using jet bins, Phys. Rev. D 85, 034011 (2012). 